\title{
Les transformations des sciences humaines et sociales en Russie
}

Transformations in human and social sciences in Russia

Las transformaciones de las ciencias humanas y sociales en Rusia

Liudmila Pipiya

Traducteur : Cécile Boyer et Michel Lussault

\section{(2) OpenEdition}

\section{Journals}

Édition électronique

URL : http://journals.openedition.org/ries/564

DOI : $10.4000 /$ ries.564

ISSN : 2261-4265

Éditeur

Centre international d'études pédagogiques

\section{Édition imprimée}

Date de publication : 1 décembre 2008

Pagination : 51-60

ISSN : 1254-4590

\section{Référence électronique}

Liudmila Pipiya, «Les transformations des sciences humaines et sociales en Russie », Revue internationale d'éducation de Sèvres [En ligne], 49 | décembre 2008, mis en ligne le 01 décembre 2011, consulté le 20 avril 2019. URL : http://journals.openedition.org/ries/564 ; DOI : 10.4000/ries.564 


\section{Les transformations des sciences humaines et sociales en Russie*}

\section{Liudmila Pipiya}

Le statut actuel des sciences humaines et sociales (SHS) ne fait pas l'objet, de manière générale, d'un examen approfondi par la communauté des chercheurs russes ni par la société russe. Il arrive parfois cependant que surviennent d'âpres discussions au sein de la communauté des SHS sur le rôle et l'avenir de celles-ci en Russie. Elles révèlent alors un large éventail de points de vue sur la recherche et sur l'enseignement en sciences humaines dans ce pays.

Cet article n'émet pas de jugement sans équivoque sur les connaissances en sciences humaines établies par les universitaires russes. Il met simplement en lumière les tendances actuelles du monde des SHS en Russie ${ }^{1}$.

\section{UNE FORTE CROISSANCE RÉCENTE DE LA RECHERCHE EN SHS EN RUSSIE}

La Russie jouit encore aujourd'hui d'un substrat scientifique conséquent ainsi que d'un système éducatif développé. Dans l'ensemble, le rôle de la science y demeure prépondérant, malgré le vieillissement des chercheurs et une fuite des cerveaux particulièrement importante dans la seconde moitié des années 1990. D’après les données statistiques de l'État, 3622 organisations prenaient part en 2006 à la recherche et au développement et $51 \%$ d'entre elles appartenaient au secteur public, notamment les établissements publics d'enseignement supérieur qui, à eux seuls, représentent $28 \%$ des organisations publiques s'adonnant à la recherche et au développement [R\&D] (Pipiya, 2007). Si l'on exclut le personnel enseignant des universités, le nombre de chercheurs en sciences humaines et sociales ne représente que $5 \%$ du nombre total de chercheurs. En 2005, on dénombrait 20800 chercheurs en SHS. La proportion de personnel enseignant spécialisé en SHS dans l'enseignement supérieur est bien plus considérable, mais les statistiques de l'État ne comprennent pas de données détaillées rendant compte des différentes disciplines.

\footnotetext{
* Article traduit de l'anglais par Cécile Boyer avec la collaboration de Michel Lussault.

1. Le contenu de cet article s'appuie sur le rapport de recherche effectué dans le cadre du projet de collaboration de recherche en sciences humaines et sociales entre l'Europe, la Russie, d'autres pays de la CEl et la Chine développé grâce au Sixième programme-cadre pour la recherche de l'Union européenne (Zavarukhin et Pipiya, 2007).
} 
À ce jour, la situation des sciences naturelles et des sciences humaines et les distinctions faites entre elles sont pratiquement l'inverse de ce qu'elles étaient il y a vingt ans, à l'époque où les sciences naturelles étaient considérées comme les seules «vraies» sciences capables d'influencer fondamentalement la vie sociale en Russie, alors que les sciences humaines étaient perçues comme des disciplines à forte teneur idéologique et de peu d'utilité. Maintenant au contraire, suite à l'abandon de nombreuses recherches fondamentales (faute de moyens) et en raison de la quasi-désertion par les chercheurs de nombreux instituts expérimentaux, on observe qu'un certain nombre de disciplines humaines et sociales sont en plein essor, avec en tête l'économie et les sciences politiques.

Pour ces disciplines, la forte croissance du nombre de centres spécialisés et de centres de recherches est parfois qualifiée «d'avalanche». Par exemple, on dénombre aujourd'hui en Russie plus de trois cents centres de sciences politiques qui ont un chiffre d'affaire annuel d'environ $\$ 300000$. Au cours des cinq dernières années, le nombre d'étudiants en troisième cycle et de doctorants a été multiplié par trois en sciences politiques et par 2,5 en économie; le nombre de mémoires soutenus a également augmenté proportionnellement (Yurevich, 2004).

Toutefois, l'accroissement du volume et de l'intérêt porté aux sciences sociales n'est pas toujours accompagné d'un gain proportionnel de qualité de la recherche. Par exemple, plus de $90 \%$ des centres de recherches sociologiques créés depuis le début des années 1990 mènent des études qui, en règle générale, s'en tiennent à collecter et répertorier des données brutes.

Début 2006, le nombre total des établissements d'enseignement supérieur était de 1068, soit 655 établissements publics et 413 établissements privés. Une grosse majorité des établissements d'enseignement supérieur privés propose des cursus spécialisés en SHS (Pipiya, 2007).

Les enquêtes auprès des étudiants montrent qu'ils considèrent l'économie, le droit, les sciences politiques, les relations internationales, la sociologie et la psychologie comme des disciplines importantes, alors que pratiquement personne ne mentionne la physique ou la chimie. Les étudiants russes divisent les SHS en trois catégories: l'économie et le droit sont les «disciplines phares", la sociologie, la psychologie et les relations internationales des «disciplines d'avenir» et l'histoire, la philologie, la psychologie, les études culturelles et les sciences de l'éducation sont rangées dans la catégorie moins prestigieuse des «disciplines mineures». Ces tendances sont identiques chez les étudiants en troisième cycle et chez les doctorants. Elles sont prépondérantes lorsque les diplômés en SHS sont employés dans les entreprises et qu'ils mènent des carrières de managers dans des compagnies privées et des corporations.

Outre les établissements d'enseignement supérieur et les organismes de recherche en SHS, il existe aussi un grand nombre d'associations et de réseaux de recherche et de nombreuses associations professionnelles spécialisées en SHS, 
comme la Société russe de philosophie, la Société russe des sociologues, l'Association russe de sciences politiques, l'Association des conseillers en économie et gestion, l'Association de l'histoire mondiale, la Société russe de l'histoire de la pensée, l'Association des études européennes, la Société d'économie libre de Russie, l'Association de recherche économique dans le secteur public, l'Association des anthropologues et des ethnologues de Russie, le Centre d'études du droit romain, l'Union russe des amis de la constitution et d'autres encore. Leur statut est tantôt russe, tantôt interrégional, c'est-à-dire concernant plusieurs républiques de l'ancienne URSS, membres de la Fédération de Russie.

\section{UNE ORGANISATION PLUS SOLIDE ET DE NOUVEAUX FINANCEMENTS}

Pendant la période de réformes, la communauté de recherche en SHS a réussi à s'organiser de manière visible et pour la première fois depuis bien longtemps. Les centres de recherches les plus prometteurs furent soutenus par des fonds provenant de sources internationales, russes et régionales. Des centaines de projets à différentes échelles furent conçus et menés à bien (ces projets allaient de la création et du développement de clubs de débats d'étudiants à la mise en place de programmes scolaires et scientifiques visant à revigorer la grande tradition scientifique de Russie et à promouvoir une éducation fondée sur des valeurs de progrès et des valeurs universelles). Un certain nombre de réseaux électroniques dévolus aux SHS ont aussi vu le jour (par exemple, le réseau électronique russe «sciences sociales et humanités» ${ }^{2}$ ), ainsi que des centres de recherches internationaux, des associations et des initiatives telles que le Centre franco-russe des SHS ou l'Association russo-américaine d'échanges académiques «des professionnels pour la coopération ${ }^{3}$ », l'Association internationale des conflictologues, etc.

En Russie, la majeure partie du soutien à la fois financier et organisationnel provient de l'étranger par le biais de programmes visant à la promotion d'une recherche en SHS indépendante. L'un des exemples les plus parlants est le Programme interrégional de recherches en sciences sociales initié par l'organisation caritative ISE-Center ${ }^{4}$ implantée en Russie en 2002. Ce programme combine la participation de la Carnegie Corporation, de la Fondation John D. and Catherine T. MacArthur, de l'Institut Kennan et du ministère de l'éducation russe faisant aujourd'hui partie du ministère de l'éducation et de la science de la Fédération russe. L'objectif de base de ce programme est de permettre l'intégration du potentiel des universités russes à la recherche en SHS. Les résultats obtenus sont à ce jour positifs.

\footnotetext{
2. Ndt: «Social Sciences and Humanities».

3. Ndt: «Professionals for the Cooperation».

4. ISE-Center : Information. Scholarship. Education (Information. Scolarité. Enseignement).
} 
Concernant le soutien des SHS, il faut souligner que la Russie contribue davantage financièrement aux processus d'élaboration de savoirs que la plupart des pays ayant un PIB/habitant similaire. Le coût total de la R\&D en Russie s'élève à environ $1,1 \%$ du PIB. La majeure partie de la R\&D russe est financée par l'État. Donc il est nécessaire de faire plus systématiquement dépendre l'attribution de financements de la compétitivité des projets. Des progrès en ce sens ont déjà été réalisés mais les choses évoluent lentement : le budget de 2006 n'attribuait que 14,6\% de tous les financements des sciences (hors recherches militaires) en fonction de critères de compétitivité. Et un peu plus de la moitié de ces financements devaient passer par la Fondation russe pour la recherche fondamentale (FRRF) et la Fondation russe des sciences humaines (FRSH). Les données sur les dépenses internes annuelles concernant les SHS par secteur montrent qu'en 2005, 42,2\% du total des dépenses courantes en sciences sociales et $78,5 \%$ de celles en sciences humaines bénéficiaient à des instituts de recherche publics et que la part consacrée à la recherche en SHS dans le cadre de l'enseignement supérieur était bien moindre : seulement $30,7 \%$ pour les sciences sociales et $19,1 \%$ pour les sciences humaines (Pipiya 2007).

\section{L'INTÉGRATION DE LA SCIENCE ET DE L'ÉDUCATION}

L'une des orientations prospectives clés de la politique en science et technologie (S\&T) de la Fédération russe consiste en la mise en place de conditions favorables à l'intégration de la science et de l'éducation, et en un fonctionnement efficace des grands organismes d'enseignement scientifique intégrés. Les autorités considèrent qu'une forte intégration, quasi organique, de la science et de l'enseignement est un facteur essentiel, déterminant pour la qualité de la croissance, de l'innovation et du potentiel économique, et, de manière plus générale, pour la sécurité nationale du pays. Cette évolution rompt avec l'héritage de la période soviétique lors de laquelle on avait plutôt séparé recherche et enseignement supérieur.

La création d'organismes intégrant science et éducation (universités de recherche, facultés dans les disciplines fondamentales, laboratoires conjoints, etc.) semble garantir une amélioration de la qualité, de l'efficacité et de la renommée de la recherche et de l'enseignement, ainsi que le renouvellement des vocations de chercheurs et d'enseignants. Le fait que les diplômés du supérieur s'impliquent dans la recherche moderne est extrêmement important pour juguler le départ des jeunes experts à l'étranger et même favoriser leur retour dans le pays.

Le principal programme-cible fédéral pour soutenir la $R \& D$, «Recherche et développement dans les secteurs prioritaires de la S\&T», comprend des projets de recherches en sciences économiques et sociales, et la 
formation de jeunes scientifiques et d'experts dans de grands centres éducatifs scientifiques (y compris des centres étrangers). Depuis ces dernières années, dans le cadre de ce programme, le président de la Fédération russe attribue, sur la base de critères de compétitivité, des bourses d'État pour soutenir les jeunes scientifiques (les doctorants en sciences et leurs tuteurs, et les jeunes docteurs en sciences). En 2006, environ six cents bourses ont été attribuées à de jeunes scientifiques doctorants ou docteurs en sciences, dont plus d'une centaine en SHS.

Dans le cadre du projet national prioritaire en matière d'éducation, le président russe a également créé plusieurs prix récompensant les jeunes talents âgés de quatorze à vingt-cinq ans. Il existe aussi un certain nombre de bourses régionales.

Les fondations décernant des bourses représentent le principal nouveau mécanisme secondant l'État dans le soutien à la recherche en SHS introduit en Russie au début de la phase de transformation économique. Avec leur implantation, une nouvelle culture s'est développée au sein de la communauté russe de chercheurs. L'attribution de ces bourses se fonde sur certains critères distinctifs comme la libre compétition pour obtenir un financement, une définition ascendante des projets de recherche et la validité des projets. C'est d'ailleurs ainsi que sont gérés les fonds similaires dans les pays occidentaux. Cependant, bien souvent, la transparence et l'ouverture à tout type de compétition, ainsi que la mise à la disposition des candidats des conclusions écrites des experts et des commissions concernant leur dossier de candidature laissent grandement à désirer.

Outre les crédits alloués par les autorités exécutives fédérales ou régionales, on trouve en Russie d'autres sources de financement des SHS, en particulier par le biais des ONG étrangères. Les fondations privées russes soutenant le développement des SHS sont trop peu nombreuses, bien que l'on note une tendance récente à l'amélioration de cette situation. Outre la FRSH, le programme présidentiel et les bourses régionales, certaines fondations d'entreprises russes et étrangères soutiennent aussi les projets en SHS (fondations Dynasty et Potanin, entre autres).

\section{UNE INTERRELATION FORTE ENTRE LES SCIENCES HUMAINES ET UNE SOCIÉTÉ EN MUTATION}

Un trait spécifique à la vie intellectuelle en Russie doit être souligné. Pendant très longtemps, les intellectuels russes s'appuyaient sur la littérature bien plus que sur les sciences sociales pour fonder leur pensée. Ce qui a été pensé au XIX ${ }^{\mathrm{e}}$ siècle grâce aux sciences sociales (économie, sociologie, etc.) dans les pays anglo-saxons a été étudié en Russie à la lumière de la littérature russe. Le fait qu'à cette époque les connaissances sociales n'aient été en Russie qu'à un stade embryonnaire ne surprend donc pas. Ce qui est regrettable, en revanche, 
c'est qu'au vu des problèmes structurels de la société russe, la situation ait peu évolué en l'espace d'un siècle.

En Russie, l'édification d'une société politique et d'un État de droit reste une tâche majeure à accomplir et un vecteur nécessaire au succès et aux résultats concluants des travaux en SHS des intellectuels, à la bonne concrétisation de leur devoir historique et à leur rôle social. Il est avéré qu'un mode de pensée détermine un mode d'action. Il est donc difficile d'imaginer que quelqu'un puisse établir une démocratie si la majorité des citoyens n'a pas un mode de pensée démocratique. Il importe donc d'instaurer un programme d'éducation vaste et à long terme concernant les mécanismes et les institutions démocratiques qui pourraient être applicables à la réalité russe, comme cela avait été fait dans l'Allemagne d'après guère et a perduré dans l'Allemagne actuelle (Magaril, 2001). Les clubs de débats, les séminaires, les tables rondes, peuvent être un apport constructif en ce sens, en contribuant à l'éducation de l'opinion publique.

Au début des années 1990, les Russes ont fait l'expérience d'une profonde transformation de leur système économique et social. Toutes les catégories sociales en ont été affectées. Aujourd'hui, les communautés sociales sont en cours de formation et dans une phase de développement dynamique. À ce jour, les SHS en Russie évoluent donc dans des conditions économiques, sociales et politiques d'une nouvelle qualité, du fait des changements sans précédents survenus au cours des dix-sept dernières années. Depuis l'effondrement de l'Union soviétique, le système scolaire mis en place avec succès sous l'ère soviétique s'est considérablement détérioré. Les SHS russes doivent à présent prendre en compte de nouveaux facteurs, qu'ils soient propres à la Russie ou mondiaux : un effondrement démographique accompagné d'un fort décroissement de la population, l'établissement d'une société de l'information, le fort niveau de dépendance à l'industrie d'extraction, etc.

À l'inverse des sciences naturelles et des disciplines techniques qui, même sur fond de transformation sociale, ont un contenu qui évolue peu, les sciences sociales et les sciences humaines sont en prise directe avec l'autoreprésentation d'une société qui définit ses objectifs propres et la place qu'elle occupe dans l'histoire. De ce fait, l'identité par et dans laquelle l'État et la société russes s'affirment et se reconnaissent ayant changé au cours des dernières années, une révolution de la pensée et de la portée des SHS et de leur enseignement s'est inévitablement produite. Cependant, ces changements se mettent en place lentement et difficilement, et la société ne les considère pas à leur juste valeur ou n'en a pas toujours suffisamment conscience. Mais les sociétés qui vivent des périodes de transition et qui sont instables parce qu'en développement ont prouvé leur capacité à générer de nouvelles idées, de nouvelles structures, de nouveaux mouvements. Il s'agit par exemple de variantes et d'alternatives possibles pour élaborer un nouveau système de service public, d'acteurs de 
société civile, de culture d'entreprise et de gestion économique rationnelle; toutes ces composantes sont nécessaires au progrès des valeurs démocratiques et essentielles pour être en mesure de remporter de nouveaux défis, notamment à l'échelle mondiale.

\section{UNE ÉVOLUTION EN APPARENCE POSITIVE...}

On peut trouver au moins deux avis opposés émis par les scientifiques russes sur l'état actuel des SHS en Russie.

Selon le premier, les SHS sont en plein essor (c'est le point de vue le plus répandu et le plus souvent exprimé par les officiels du gouvernement fédéral, les directeurs de facultés en SHS, les professeurs de chaire, les instituts de recherche, les entreprises privées, les entreprises commerciales de services et les institutions politiques). Leurs arguments pour étayer cette opinion sont relativement simples et s'inscrivent dans une logique «linéaire» de croissance soutenue.

Sans occulter cet aspect purement quantitatif du processus de croissance, il faut tout de même souligner que les estimations relevant de ce point de vue ne tiennent pas compte, à bien des égards, de la qualité du contenu de la recherche et de l'enseignement russes des SHS. Les chiffres illustrant la croissance soutenue des SHS en Russie, n'apportent aucune preuve d'un gain de qualité significatif. Ce constat nourrit un second point de vue, plus critique.

Les tendances suivies par les SHS dans la période postsoviétique sont donc contradictoires. Pour chaque pas en avant, il y a trois pas en arrière. C'est probablement dû au fait que la nouvelle situation dans laquelle ces sciences s'inscrivent comporte en elle-même des contradictions. C'est pourquoi les points de vue divergent. Cette nouvelle situation découle naturellement des transformations de la société russe moderne, et les sciences humaines et sociales russes doivent se confronter à ces véritables épreuves. Une formule universelle exprime cette idée : la qualité d'une société se transmet à ses sciences humaines et sociales.

Reste qu'on ne peut pas nier que les SHS sont soumises à la pression de leur environnement social. On peut probablement au moins souligner deux problèmes auxquels les sciences humaines sont confrontées dans la situation russe.

Le premier est un manque de connaissances des processus réellement à l'œuvre dans la société russe. En Russie, les mécanismes sociaux sont de plus en plus obscurs même si les autorités entretiennent l'illusion d'une transparence de l'information dans la société. L'absence de transparence et le refus de délivrer des informations objectives restreignent drastiquement le champ d'application des sciences sociales, et, cela est crucial, le besoin qu'on en a. Il en résulte que 
la communauté des SHS travaille souvent en s'appuyant plus sur des mythes et des spéculations que sur des faits et des phénomènes avérés scientifiquement.

Le second est l'effet des délais en constante augmentation. Il y a en effet un décalage entre la situation réelle de la société russe et la retranscription de cette situation dans les SHS, qui s'explique par le fait que les dynamiques sociales en Russie vont si vite qu'il est difficile de les étudier en temps réel. La stabilité de la société avant les réformes facilitait son étude et les sciences sociales pouvaient en faire des "photos» (même si à l'époque, il était aussi extrêmement difficile de mener des recherches objectives sur la société). Ayant des ressources limitées, les spécialistes de sciences humaines et sociales ne peuvent exploiter tout leur potentiel pour étudier cette dynamique de changement en temps réel.

Les facteurs précédemment mentionnés affectent aussi la qualité de l'enseignement des SHS. Pourtant, l'on peut constater qu'une nette amélioration des caractéristiques de base de la société influe sur les SHS russes. En comparaison de l'époque soviétique, la communauté russe des SHS jouit aujourd'hui des «quatre libertés»: liberté d'organiser et de planifier la recherche; liberté de communiquer et d'échanger avec l'étranger; liberté d'exprimer n'importe quelle idée ou concept scientifique; liberté de collecter et d'utiliser des informations. Toutefois, d'une certaine manière, ces libertés demeurent accordées plus en théorie qu'en pratique.

\section{... MAIS DES fACTEURS DE CRISE}

Durant ces dernières années, de nouvelles formes d'interaction entre les SHS et la société se sont révélées. Si les premières années de transformation sociale ne remettaient pas en cause l'importance des connaissances en sciences humaines et sociales et qu'il s'agissait alors seulement de trouver le moyen de les maîtriser et de les développer au mieux, un certain scepticisme se manifeste à présent concernant le développement des SHS. Dans les médias de masse, on peut souvent entendre les déclarations d'experts de tous types affirmant que les sciences sociales ne remplissent pas leur tâche de résolution des problèmes de la Russie, qu'elles s'avèrent inefficaces, et qu'elles sont, en quelque sorte, des sciences strictement occidentales inapplicables à la situation en Russie qui requiert une approche et des techniques scientifiques spécifiques. Ces déclarations sous-entendent que les SHS sont incapables de devenir l'instrument de mesure scientifique des réformes russes.

Dans un tel contexte, il est important de rappeler que tant du point de vue des faits avérés que de l'image que la Russie se fait d'elle-même aux plans national, économique et social, le pays a connu d'énormes bouleversements en l'espace de quelques années et se retrouve confronté à de graves problèmes par manque d'expérience pour trouver des solutions adaptées. Les SHS russes, qui sortent à peine des "catacombes de la période soviétique», se sont directement 
retrouvées plongées dans une situation de marché de la science et de la formation (ou plutôt, dans les cas des SHS, d'une quasi-situation de marché). Comparé à celui des sociétés occidentales plus stables, le marché russe (qui inclut le marché de l'expertise scientifique et des services d'enseignement) est plus rigide, plus anti-intellectuel et moins institutionnalisé. S'y adapter suppose souvent que les chercheurs renoncent à leurs principes professionnels et se plient ainsi complètement aux exigences des «clients». L'orientation choisie par nombre d'établissements de recherche et d'enseignement, qui tentent d'atteindre une position confortable et une certaine aisance par cette stratégie, illustre ce propos.

Un phénomène plutôt nouveau (ou plus exactement qu'on a eu tendance à occulter pendant longtemps) consiste à vouloir affilier les SHS russes à des catégories idéologiques. Il n'y a pas en principe d'idéologie officielle en Russie aujourd'hui. Cependant, les tentatives d'en instaurer une s'expriment en permanence sous de multiples formes. Les apologètes s'immiscent dans le monde des SHS et tiennent le devant de la scène. De nombreux universitaires spécialistes des SHS se transforment en maîtres à penser, en prêcheurs sociaux s'exprimant au nom de toute la société et sachant de manière certaine ce qu'il faut faire pour développer la Russie. Dans un même mouvement, un constructivisme social, l'avènement de mentors et un dédain pour une analyse concrète de la société s'articulent, passant complètement à côté de la nécessité de développer une expertise scientifique indépendante de la vie sociale sous tous leurs aspects et des tensions politiques. Il devient parfois difficile de savoir où s'arrête le discours scientifique et où commence le discours politique dans les publications et les programmes d'enseignement des SHS. Le prêt-à-penser prend le pas sur la discussion scientifique objective et indépendante, dotée d'un réel contenu.

Outre ces éléments spécifiques altérant la «bonne santé» des SHS en Russie et préoccupant, pour le moins, la communauté scientifique, il faut aussi aborder les relations qu'elles entretiennent avec les sciences en Occident.

L'influence de l'Occident (et non l'influence d'une internationalisation éclairée) s'est fait sentir par le biais de directives imposées par un certain nombre de fondations scientifiques internationales qui n'apportaient leur soutien financier qu'aux recherches et programmes d'enseignement répondant aux attentes d'un mode de penser unifié, s'inscrivant dans la mondialisation, sans se soucier des problématiques propres à la société russe. Cela a conduit à l'émergence d'un petit groupe de bénéficiaires de subventions attribuées par ces fondations occidentales implantées en Russie. Ces bénéficiaires n’ont pas vraiment de grand potentiel scientifique mais ils savent se maintenir dans l'air du temps. Ils passent d'un programme à un autre et en retirent un bénéfice considérable. Ainsi, les programmes d'enseignement de bien des établissements supérieurs et les publications de bien des chercheurs russes usent et abusent de constructions de concepts et d'une terminologie qui ne sont guère plus qu'un copier-coller de sources occidentales. Par ailleurs, les auteurs de ces 
publications et de ces programmes n'ont pas de connaissance approfondie de l'essence théorique des bases conceptuelles qu'ils utilisent.

Les financements étrangers ont beaucoup soutenu et soutiennent encore les SHS en Russie. Mais l'utilisation de programmes de soutien étrangers conduisant à un recyclage intellectuel effectué par un personnel scientifique médiocre, sous prétexte de répondre aux exigences du moment, ne fait pas honneur à la communauté scientifique russe.

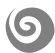

En comparant le premier point de vue «linéaire» et serein sur l'état des SHS russes et le second plutôt alarmiste, l'analyse qui s'impose est que tout ne va pas si bien qu'il y paraît pour les SHS en Russie. Tout au moins, il ne faut pas s'arrêter naïvement à la seule croissance en volume de la recherche en SHS. En Russie, les SHS modernes sont en ce moment soumises à une dynamique de transformation qui leur fait emprunter un cheminement parfois douloureux.

Il est nécessaire d'examiner de manière approfondie en quoi les connaissances en sciences humaines contribuent aux prises de décisions dans le secteur public. Mais d'un point de vue général, il est possible d'affirmer de manière à peu près certaine que, dans la Russie moderne, les sciences sociales n'ont pas réussi à définir de formes d'organisation politique et économique susceptibles de résoudre la complexité des problèmes auxquels la société est en proie. Jusqu'en ce début de $\mathrm{XXI}^{\mathrm{e}}$ siècle, la Russie a semblé incapable de trouver les réponses dont elle a besoin pour relever ce grand défi historique : créer une économie capable de produire tout ce qui est nécessaire à la vie d'une société de cette taille; élaborer un système politique permettant l'établissement d'une économie efficace; former une masse critique d'élite dotée de qualités intellectuelles et morales indéniables. Ce qui se passe aujourd'hui en Russie témoigne de manière convaincante du fait que les sciences naturelles, les sciences de l'ingénieur, l'enseignement musical et le ballet, même à haut niveau, ne suffisent pas pour comprendre et construire une société démocratique.

\section{BibLIOgRAPHIE}

MAGARI S.A. (2001) : Civil Liability of Intellectuals. Sociological Studies, $\mathrm{n}^{\circ} 2$, pp. 51-57 [en russe].

PIPIYA L. (2007) : Measuring SSH Potential. GlobalSSH Data Book, Moscow : CSDS RAS.

YUREVICH A. V. (2004) : Social and Humanitarian Science in Contemporary Russia : Adaptation to a Social Context. Working paper, Moscow : SU-HSE [en russe].

ZAVARUKHIN V. and PIPIYA L. (2007) : Institutional Landscape and Research Policy for SSH in Russia. Institutional report for Russia. GlobalSSH project, [http://www.globalsocialscience.org]. 